\title{
Ogilvie syndrome: acute pseudo-obstruction of the colon
}

\author{
Huan-Lun Hsu MD, Yao-Ming Wu MD PhD, Kao-Lang Liu MD
}

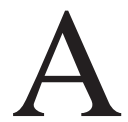
76-year-old man with a history of hepatitis C infection and newly diagnosed hepatocellular carcinoma was admitted for scheduled trans catheter arterial chemoembolization therapy. He developed abdominal discomfort and nausea one day after the procedure. On examination, he had a distended abdomen, diffuse tenderness without rebound pain and reduced bowel sounds. He was afebrile, and results of blood tests were unremarkable. Plain abdominal radiography showed diffuse bowel loop dilation (Figure 1A), and computed tomography showed marked bowel loop dilation and colonic gas, without obstructive masses or ischemic bowel lesions (Figure 1B).

We diagnosed acute pseudo-obstruction of the colon (Ogilvie syndrome). Nasogastric decompression and discontinuation of oral intake did not relieve our patient's abdominal pain. He developed dyspnea because of persistent abdominal distention, and we decided to use colonoscopic decompression followed by neostigmine. He recovered fully from the syndrome and received treatment for hepatocellular carcinoma.

Ogilvie syndrome is marked by nonmechanical dilation of the cecum and right colon. ${ }^{1}$ It presents with abdominal pain and abnormal intestinal motility, and is not uncommon in patients with critical illnesses, electrolyte imbalances, anticholinergic medication regimens and recent surgery. In our patient, we believe the syndrome was related to trans catheter arterial chemoembolization therapy. The exact pathogenesis of the syndrome remains unclear, although imbalance of the autonomic nervous system affecting the colon has been proposed. ${ }^{2}$

\footnotetext{
From the Department of Internal Medicine (Hsu), China Medical University Hospital, Taipei Branch, and the Departments of Surgery (Wu) and Medical Imaging (Liu), National Taiwan University Hospital, National Taiwan University, College of Medicine, Taipei 100, Taiwan

CMAJ 2010. DOI:10.1503/cmaj.100200
}

Unlike toxic megacolon (often caused by inflammatory bowel disease or infection), Ogilvie syndrome usually does not present with signs of systemic toxicity. Computed tomography is usually indicated for ruling out obstructive lesions or possible bowel ischemia. If the syndrome is left untreated, life-threatening complications such as bowel ischemia or perforation may occur in an estimated 3\%-15\% of patients, with a $50 \%$ reported mortality rate. ${ }^{2}$ The success rate for nasogastric decompression with or without anal tube placement is variable (20\%-92\%), and colonoscopic decompression is successful in about $70 \%$ of patients. ${ }^{2}$ A resolution rate of $80 \%$ $90 \%$ has been reported for use of neostigmine $(2.0 \mathrm{mg}$ intravenously) when conservative therapy has failed. ${ }^{2}$ Surgical management with cecostomy or colectomy is reserved for refractory cases.

This article has been peer reviewed.

Competing interests: None declared.

\section{REFERENCES}

1. Pratt DS, Epstein SK. Recent advances in critical care gastroenterology. Am J Respir Crit Care Med 2000;161:1417-20.

2. Eisen GM, Baron TH, Dominitz JA, et al. Acute colonic pseudo-obstruction. Gastrointest Endosc 2002;56:789-92. 